\title{
Use of calculus of variations to determine the shape of hovering rotors of minimum power and its application to micro air vehicles
}

\author{
O Lopez-Garcia ${ }^{1 *}$, A Cuerva ${ }^{1}$, and S Esteban ${ }^{2}$ \\ ${ }^{1}$ Instituto Ignacio Da Riva, Universidad Politécnica de Madrid, Madrid, Spain \\ ${ }^{2}$ Department of Aerospace Engineering. Universidad de Sevilla, Sevilla, Spain
}

\begin{abstract}
In this paper, calculus of variations and combined blade element and momentum theory (BEMT) are used to demonstrate that, in hover, when neither root nor tip losses are considered; the rotor, which minimizes the total power (MPR), generates an induced velocity that varies linearly along the blade span. The angle of attack of every blade element is constant and equal to its optimum value. The traditional ideal twist (ITR) and optimum (OR) rotors are revisited in the context of this variational framework. Two more optimum rotors are obtained considering root and tip losses, the ORL, and the MPRL. A comparison between these five rotors is presented and discussed. The MPR and MPRL present a remarkable saving of power for low values of both thrust coefficient and maximum aerodynamic efficiency. The result obtained can be exploited to improve the aerodynamic behaviour of rotary wing micro air vehicles (MAV). A comparison with experimental results obtained from the literature is presented.
\end{abstract}

Keywords: micro air vehicles, low Reynolds number aerodynamics, calculus of variations, hovering rotor, aerodynamic optimization, blade element momentum theory

\section{INTRODUCTION}

The increasing interest and efforts devoted to the development of smaller, lighter, and more agile micro air vehicles is principally focused on fixed, rotary, and flapping wing vehicles. According to reference [1] micro air vehicles (MAV) sometimes are also referred to as nano air vehicles (NAV) and the frontier between both is not well established. Their maximum gross take-off weight is less than $0.2 \mathrm{~kg}$ and length dimensions are less than $0.15 \mathrm{~m}$. In what follows, and because this paper is focused on rotary wing vehicles, the acronym MAV will apply to rotary

* Corresponding author: Instituto Ignacio Da Riva, Universidad Politécnica de Madrid, Plaza Cardenal de Cisneros 3, 28040, Madrid, Spain.

email: oscar.lopez.garcia@upm.es wing micro or nano air vehicles. Rotary wing vehicles are particularly well suited for reconnaissance or observation missions because they excel at hovering, which is the main flight mode of such missions. However, aerodynamic forces depend on Reynolds number, and it is very well known that MAV operate at very low Reynolds numbers (see for instance reference [2]) at which the aerodynamic efficiency severely decreases. Taking this into consideration, the maximization of hover performance of MAV necessarily requires the optimization of the rotor design. A literature review of the state of MAV developments and emerging technological trends oriented to improve the flight efficiency of these vehicles can be found in reference [1].

Helicopter design optimization is a complex issue because, among other aspects, the optimization process usually involves multidisciplinary objectives 
together with an important number of constraints. A literature revision of helicopter optimization was presented in reference [3] and the following optimization problems were reviewed: composite rotors, detailed geometry shape, flight mechanics, aeromechanical stability, tilt rotor applications, and smart rotor. However, the optimization of MAV is not specifically addressed in this reference. The aerodynamic shape optimization of a rotorcraft has been carried out using different aerodynamic modelling of the main rotor. Thus non-linear frequency domain method to solve the unsteady viscous flow is applied in reference $[4]$ and a 3D Navier-Stokes solver is used in reference [5]. Other authors used simpler models, for instance a combined lifting-line blade with a prescribed wake and a free-wake models are applied in reference $[6]$. In some cases, even the classic combined blade element and momentum theory (BEMT) is used to perform an aerodynamic shape optimization of a coaxial rotor, see reference [7], or to evaluate new rotor control mechanisms in MAV, see reference [8]. An example of the utilization of the simplest aerodynamic models, Rankine-Froude momentum theory, is applied to predict helicopter performance in reference $[\mathbf{9}]$.

Because MAV have their own particularities, optimization problems involving such vehicles face different challenges. One difficulty is the manufacturing process for blades of such a small span and chord. Another is that experimental data for airfoils at low Reynolds numbers is difficult to source. The analysis of the performance of a low $\left(4 \times 10^{3}\right)$ Reynolds number airfoil $[\mathbf{1 0}]$ indicates that with decreasing Reynolds number the profile thickness needs to be reduced in order to maintain performance characteristics. Among other considerations, these aspects have led researchers to focus on the development of prototypes based on simplistic models to reduce computational burden and hence enable efficient optimization studies to be conducted. Therefore, in most cases, aerodynamic design and optimization of MAV have been based on the BEMT despite the fact that researchers are aware of the limitations of such an approach, as is pointed out in reference [1]. For instance, small rotor design optimization using BEMT has been recently studied in reference $[\mathbf{1 1}]$. In this investigation, circular arc airfoils were used and low Reynolds numbers in a range from $5 \times 10^{3}$ to $6 \times 10^{4}$ were considered. It is well known that at these low Reynolds numbers, the aerodynamic efficiency of a conventional airfoil is reduced in comparison to that at high Reynolds numbers $[12,13$. The review presented in reference $[\mathbf{1 4}]$ remains the basic reference to describe the aerodynamics of low Reynolds numbers airfoil. Recently, some efforts to experimentally understand the behaviour of the wake of a rotor at low Reynolds numbers have been carried out. For instance, a detailed high-resolution flow visualization of the viscous wake of a rotor operating at low Reynolds numbers can be seen in reference [15]. The development of a micro-coaxial rotorcraft and its hovering performance are described in reference [16]. Moreover, because the small size of the blades plays a fundamental role in the manufacturing of the rotor, many of the airfoils used are of a relatively simple shape, for instance the above-mentioned circular arcs, as presented in reference $[\mathbf{1 1}]$. These simple aerodynamic shapes exhibit low aerodynamic efficiency, thus further emphasizing the need for designing optimum low Reynolds number rotors.

The goal of this article is to provide a mathematical framework based on calculus of variations to obtain the geometry of the minimum power rotor under the classic combined BEMT. Once this framework is established, the rotor which minimizes power coefficient, for a given thrust coefficient, emerges as a natural consequence of the mathematical theory. This minimum power coefficient rotor (MPR) does not correspond to the traditional ideal twist rotor (ITR) or the traditional optimum rotor (OR) defined by most helicopter textbooks.

Another contribution of this study is to show that the MPR tends to the OR when the aerodynamic efficiency is high. If the argument is reversed, it can be concluded that for low aerodynamic efficiencies the difference between the power required in hover by the MPR and the OR can be extremely important. This result could play a fundamental role in the aerodynamic design of MAV because the aerodynamic efficiency of such aircrafts is low enough to underline the practical interest of this result.

Finally, the analysis of optimum blade geometries and the corresponding performances including root and tip losses is undertaken. This study reveals that considering root and tip losses modifies significantly the optimum shapes at the blade extremes leading to a better manufacturability but worsens the performances, as expected.

\section{FORMULATION OF THE OPTIMIZATION PROBLEM}

This section is divided into two parts. First, the BEMT that describes the aerodynamic power consumption of the rotor is briefly reviewed. Second, the application of calculus of variations is used to define a family of power-related optimization problems. 


\subsection{Blade element momentum theory}

As mentioned above, the aerodynamics of a rotor can be described using quite different mathematical models. These models range from Navier-Stokes solvers at the high end of complexity through to blade element and momentum theories at the low level. Blade element theory considers the rotor divided into aerodynamically independent annular rings. The aerodynamic state of every ring is completely defined when the induced velocity is determined. Momentum theory provides a global description of the aerodynamic state of the rotor (actuator disk). Applying the momentum balance to a differential ring of the stream tube of the rotor, a local version of the momentum theory is obtained, giving rise to the well-known Froude-Finsterwalder equation, see reference $[17]$. A combination of both differential momentum and blade element theories is established on the basis that the differential thrust computed by both theories must be equal. Following the classic textbooks $[18,19,20]$, the resulting equations are linearized. Finally, the dimensionless expression of the equality of differential thrust is

$$
\frac{\sigma}{2} x^{2} C_{l}(\alpha)-4 x \lambda_{i}^{2} F\left(\lambda_{i}\right)=0
$$

where $\sigma$ is the rotor local solidity, $x$ the dimensionless position along the blade length, $\alpha$ the angle of attack, $C_{l}$ the lift coefficient, $\lambda_{i}$ the dimensionless induced velocity (positive for upwash), and $F\left(\lambda_{i}\right)$ is Prandtl's root and tip loss function expressed by

$$
\begin{aligned}
F\left(\lambda_{i}\right)= & \left(\frac{2}{\pi}\right)^{2} \cos ^{-1}\left[\exp \left(-\frac{b}{2} \frac{x-x_{R}}{\lambda_{i}}\right)\right] \\
& \times \cos ^{-1}\left[\exp \left(-\frac{b}{2} \frac{1-x}{\lambda_{i}}\right)\right]
\end{aligned}
$$

where $b$ is the number of blades and $x_{R}$ is the dimensionless root cutout (see reference $[\mathbf{2 0}]$ ).

The angle of attack is related to the blade section pitch angle, $\theta$, through the expression $\alpha=\theta+\lambda_{i} / x$. Using these linearized equations the thrust coefficient, $C_{T}$, the induced power, $C_{Q_{i}}$, and the profile power, $C_{Q_{0}}$, coefficients are expressed by

$$
\begin{aligned}
C_{T} & =\int_{x_{R}}^{1} \frac{\sigma}{2} x^{2} C_{l}(\alpha) \mathrm{d} x \\
C_{Q_{i}} & =-\int_{x_{R}}^{1} \lambda_{i} \frac{\sigma}{2} x^{2} C_{l}(\alpha) \mathrm{d} x
\end{aligned}
$$

and

$$
C_{Q_{0}}=\int_{x_{R}}^{1} \frac{\sigma}{2} x^{3} C_{d}(\alpha) \mathrm{d} x
$$

where $C_{d}$ is the drag coefficient. Once the shape and aerodynamic characteristics of a rotor are defined, that is, solidity, pitch angle, lift, and drag coefficients, the BEMT equation (1) yields the induced velocity radial distribution, $\lambda_{i}(x)$, and using equations (3), (4), and (5) the global coefficients $C_{T}, C_{Q_{i}}$, and $C_{Q_{0}}$ are computed.

\subsection{Variational framework}

Following the well-known theory of calculus of variations, see reference $[\mathbf{2 1}]$, let us consider the following family of optimization problems: determine the set of control functions $X_{i}(x), i=1, \ldots, n$, such that minimizes the functional

$$
J\left[X_{i}\right]=\int_{x_{R}}^{1} L\left(X_{i}, x\right) \mathrm{d} x
$$

subject to the constraint of a given thrust coefficient, $\bar{C}_{T}$

$$
\bar{C}_{T}=\int_{X_{R}}^{1} M\left(X_{i}, x\right) \mathrm{d} x
$$

being

$$
M\left(X_{i}, x\right)=\frac{\sigma}{2} x^{2} C_{l}(\alpha)
$$

and the algebraic constraint imposed by equation (1) at each annular ring

$$
N\left(X_{i}, x\right)=\frac{\sigma}{2} x^{2} C_{l}(\alpha)-4 x F\left(\lambda_{i}\right) \lambda_{i}^{2}=0
$$

where $L\left(X_{i}, x\right)$ is the objective function which has usually been related to some part of the power consumption of the rotor, for instance induced one, profile one, or other combinations.

Depending upon the optimization problem, the control functions $X_{i}$ are usually related to the shape of the blade required to optimize the objective function and the radial distributions of certain aerodynamic parameters. In our case, these control functions could be the dimensionless induced velocity, $\lambda_{i}(x)$, the pitch angle, $\theta(x)$, and rotor solidity, $\sigma(x)$; although in the following analysis, the pitch angle is substituted by the angle of attack $\alpha(x)$ of the blade elements. BEMT considers the aerodynamic state of a blade element independently of other blade elements - in other words there are no differential equations that govern the evolution of the variables along the blade span - and as such there are no state variables in this type of variational problem (for a more detailed discussion, see reference [21]).

Using standard calculus of variations, the problem is transformed by changing the integral constraint (7) to the following differential constraint 


$$
\frac{\mathrm{d} C_{T}}{\mathrm{~d} x}=M\left(X_{i}, x\right) ; C_{T}\left(x_{R}\right)=0, C_{T}(1)=\bar{C}_{T}
$$

and by adjoining the differential and algebraic constraints with their corresponding Lagrange multipliers, $\mu$ and $v$, the following augmented problem is defined

$$
\begin{aligned}
J^{*}\left[X_{i}\right]= & \int_{x_{R}}^{1}\left\{L\left(X_{i}, x\right)+\mu\left[M\left(X_{i}, x\right)-\frac{\mathrm{d} C_{T}}{\mathrm{~d} x}\right]\right. \\
& \left.+v N\left(X_{i}, x\right)\right\} \mathrm{d} x
\end{aligned}
$$

with $N\left(X_{i}, x\right)$ given by equation (9). The variational Hamiltonian of the augmented problem is defined by: $H\left(X_{i}, \mu, v, x\right)=L\left(X_{i}, x\right)+\mu M\left(X_{i}, x\right)+v N\left(X_{i}, x\right)$, and by integrating the term $\mu \mathrm{d} C_{T} / \mathrm{d} x$ by parts, the augmented problem can be written as

$$
\begin{aligned}
J^{*}\left[X_{i}\right]= & -\mu(1) C_{T}(1)+\mu\left(x_{R}\right) C_{T}\left(x_{R}\right) \\
& +\int_{x_{R}}^{1}\left[H\left(X_{i}, \mu, v, x\right)+\frac{\mathrm{d} \mu}{\mathrm{d} x} C_{T}\right] \mathrm{d} x
\end{aligned}
$$

The first variation of the augmented functional, $\delta J^{*}$, where $\delta$ stands for variation, can be written as

$$
\begin{aligned}
\delta J^{*}= & -\left.\left(\mu \delta C_{T}\right)\right|_{x=1}+\left.\left(\mu \delta C_{T}\right)\right|_{x=x_{R}} \\
& +\int_{x_{R}}^{1}\left[\left(\frac{\partial H}{\partial C_{T}}+\frac{\mathrm{d} \mu}{\mathrm{d} x}\right) \delta C_{T}+\frac{\partial H}{\partial X_{i}} \delta X_{i}\right] \mathrm{d} x
\end{aligned}
$$

To minimize the functional $J^{*}$, its first variation must be equal to zero, that is, $\delta J^{*}=0$. This can only happen if the set of Euler-Lagrange equations of the calculus of variations are fulfilled, that is

$$
\begin{aligned}
& \frac{\mathrm{d} \mu}{\mathrm{d} x}=-\frac{\partial H}{\partial C_{T}} \\
& \frac{\partial H}{\partial X_{i}}=0, \quad i=1, \ldots, n
\end{aligned}
$$

together with the fact that the variations of the thrust coefficient at the boundary conditions, $\left.\delta C_{T}\right|_{x=1}$ and $\left.\delta C_{T}\right|_{x=x_{R}}$, are both zero because the values at the boundaries, that is $C_{T}\left(x_{R}\right)=0$ and $C_{T}(1)=\bar{C}_{T}$, are both prescribed in equation (10).

Since $\partial H / \partial C_{T}=0$, equation (14) yields a constant value for the Lagrange multiplier $\mu$. Finally, the EulerLagrange equations together with both differential and algebraic constraints, yield the two-point boundary-value problem defined by equations (9) (10), and the set of $n$ equations

$$
\frac{\partial L}{\partial X_{i}}+\mu \frac{\partial M}{\partial X_{i}}+\nu \frac{\partial N}{\partial X_{i}}=0, \quad i=1 \ldots n
$$

where $L$ depends on the particular optimization problem that is considered, but $M$ and $N$ are given by equations (8) and (9) for all of the optimization problems analysed in this paper. This two-point boundary-value problem provides $n+2$ equations to solve the $n$ optimum control functions, $X_{i}(x)$, and the two Lagrange multipliers $\mu$ and $\nu(x)$. For the more general case considered here, the control functions $X_{i}$ in (16) are the radial distribution of induced velocity, $X_{1}=\lambda_{i}(x)$, the radial distribution of angle of attack, $X_{2}=\alpha(x)$, and the radial distribution of local solidity, $X_{3}=\sigma(x)$. Considering these definitions, equations (16) are particularized as

$$
\begin{aligned}
& \frac{\partial L}{\partial \lambda_{i}}+4 x \lambda_{i} v\left[2 F\left(\lambda_{i}\right)+\lambda_{i} \frac{\mathrm{d} F\left(\lambda_{i}\right)}{\mathrm{d} \lambda_{i}}\right]=0 \\
& \frac{\partial L}{\partial \alpha}+\frac{1}{2} \sigma x^{2}(\mu-v) \frac{\mathrm{d} C_{l}(\alpha)}{\mathrm{d} \alpha}=0 \\
& \frac{\partial L}{\partial \sigma}+\frac{1}{2} x^{2}(\mu-v) C_{l}(\alpha)=0
\end{aligned}
$$

with $F\left(\lambda_{i}\right)$ given by equation (2).

\section{DETERMINATION OF OPTIMAL HOVERING ROTORS}

In what follows, the mathematical formulation presented in section 2 is applied to obtain the characteristics of the ideal twist rotor, ITR, the optimum rotor, $\mathrm{OR}$, and the minimum power coefficient rotor, MPR. Finally root and tip losses are included in the optimization process, and two additional optimum rotors are obtained, the ORL and MPRL, where L stands for 'root-tip losses included'.

\subsection{Ideal twist rotor without root-tip losses}

The traditional ITR is defined as the rotor which minimizes the induced power coefficient $C_{Q_{i}}$, for a given thrust coefficient $\bar{C}_{T}$, and for constant solidity, $\sigma$ (see reference $[\mathbf{1 9}]$ ). In this case the objective function $L$ is

$$
L=-\lambda_{i} \frac{\sigma}{2} x^{2} C_{l}(\alpha)
$$

Only two control functions, $X_{1}=\lambda_{i}(x)$ and $X_{2}=\alpha(x)$, are considered, since the rotor local solidity is by definition constant. In the ITR analysis, neither root nor tip losses are considered, and therefore $F\left(\lambda_{i}\right)=1$ and $\mathrm{d} F\left(\lambda_{j}\right) / \mathrm{d} \lambda_{i}=0$ in equation (17). Observe that equation (19) does not apply here since $\sigma$ is constant.

Taking into account equation (20), expression (18) is fulfilled whenever $\mu-v-\lambda_{i}=0$ and/or $C_{l, \alpha}=\mathrm{d} C_{l} /$ $\mathrm{d} \alpha=0$. Since in the analysis of the ITR, linear aerodynamics is considered, the angle of attack is assumed to be below the value corresponding to maximum $C_{l}$. Therefore $C_{l, \alpha} \neq 0$ and the chosen solution for (18) is $\mu-v-\lambda_{i}=0$. The Lagrange multiplier $\mu$ can be written as 


$$
\mu=\frac{3}{2} \sqrt{\frac{\bar{C}_{T}}{2\left(1-x_{R}^{2}\right)}}
$$

and the induced velocity is

$$
\lambda_{i}=-\sqrt{\frac{\bar{C}_{T}}{2\left(1-x_{R}^{2}\right)}}
$$

which, as is well known, is independent of the radial position $x$.

In what follows, the usual analysis included in most common helicopter text books is applied to obtain the geometrical characteristics of the rotor as well as the induced and profile power coefficients. These parameters will be used later on for comparison purposes. The angle of attack is $\alpha=4 \bar{C}_{T}\left[\left(1-x_{R}^{2}\right) \sigma C_{l, \alpha} x\right]^{-1}$ and taking into account that $\theta(x)=\alpha(x)-\lambda_{i} / x$, the pitch angle distribution of the ITR is obtained as

$$
\theta(x)=\left[\frac{4 \bar{C}_{T}}{\sigma C_{l, \alpha}\left(1-x_{R}^{2}\right)}+\sqrt{\frac{\bar{C}_{T}}{2\left(1-x_{R}^{2}\right)}}\right] \frac{1}{x}
$$

Then, considering that the drag coefficient can be expressed as $C_{d}=\delta_{0}+\delta_{1} \alpha+\delta_{2} \alpha^{2}$, by using equations (4) and (5), the induced and profile power coefficients are expressed as functions of thrust coefficient by

$$
C_{Q_{i}}=\sqrt{\frac{\bar{C}_{T}^{3}}{2\left(1-x_{R}^{2}\right)}}
$$

and

$$
C_{Q_{0}}=\frac{\sigma \delta_{0}}{8}\left[1-x_{R}^{4}+\frac{4}{3} \frac{\delta_{1}}{\delta_{0}}\left(1-x_{R}^{3}\right) \alpha_{t}+2 \frac{\delta_{2}}{\delta_{0}}\left(1-x_{R}^{2}\right) \alpha_{t}^{2}\right]
$$

where $\alpha_{t}$ is the angle of attack at the blade tip, i.e. $\alpha=4 \bar{C}_{T}\left[\left(1-x_{R}^{2}\right) \sigma C_{l, \alpha}\right]^{-1}$. Therefore, the traditional result for the ITR is reproduced. Observe that $\alpha_{t}$ and $\sigma$ must satisfy this relation for the rotor to provide the given $\bar{C}_{T}$. Particularizing expressions (22), (23), (24), and (25) for $x_{R}=0$, the usual formulation of the induced velocity, the pitch angle and the induced and profile power coefficients of an ITR are obtained, when neither root nor tip losses are considered in the optimization.

\subsection{Optimum rotor without root-tip losses}

The traditional OR is defined as the rotor that presents minimum induced power coefficient and, superimposed to this condition, also presents minimum profile power coefficient (see reference [19]). The variational problem associated to this definition must then be stated in two steps. First, the minimization problem of $C_{Q_{i}}$ and second, the minimization of $C_{Q_{0}}$ taking into account the relations obtained for $\lambda_{i}(x), \quad \alpha(x)$ and $\sigma(x)$ in the minimization of the induced power coefficient (first step).

On the one hand, the control functions in this problem are $X_{1}(x)=\lambda_{i}(x), X_{2}(x)=\alpha(x)$, and $X_{3}(x)=$ $\sigma(x)$; and on the other hand, the function $L$ is identical to equation (20) but now $\sigma(x)$ is a control function. This fact makes it necessary to consider equation (19) in the problem.

Provided that neither $C_{l}(\alpha)$ nor $\mathrm{d} C_{l}(\alpha) / \mathrm{d} \alpha$ are zero, the solutions $v=\mu-\lambda_{i}$ and $\lambda_{i}=2 \mu / 3$ are obtained (observe that it has been established in section 2.2 that the Lagrange multiplier $\mu$ must be constant). Therefore, the well-known condition of constant induced velocity for arbitrary minimum induced power rotors in hover is obtained. The exact value for $\lambda_{i}$ is again the result for the induced velocity given by equation (22). On the other hand, equation (9) can be rewritten as

$$
x \sigma C_{l}(\alpha)=8 \lambda_{i}^{2}=4 \bar{C}_{T}\left(1-x_{R}^{2}\right)^{-1}
$$

which is the well-known relation for minimum induced power rotors in hover (see reference [19]). Observe that this condition leads to the ITR when constant solidity and linear dependence for $C_{l}(\alpha)$ versus $\alpha$ is assumed. However, for an arbitrary minimum induced power rotor, no final conclusion can be stated separately for $\sigma(x)$ or $\alpha(x)$ radial distributions, except that they must satisfy equation (26).

When stating the variational problem of minimization of the profile power coefficient, $C_{Q_{0}}$, relation (26) establishes a dependence between the two remaining control functions in the problem $(\sigma(x)$ and $\alpha(x)$, observe that the third one, $\lambda_{i}(x)$, has been already determined in the first step). Therefore equation (26) must be used to redefine the minimization problem of $C_{Q_{0}}$ in terms of only one control function. In this case $X_{2}(x)=\alpha(x)$ is selected. The functional is now given by

$$
\begin{aligned}
J[\alpha(x)] & =-\int_{x_{R}}^{1} \frac{\sigma}{2} x^{3} C_{d}(\alpha) \mathrm{d} x \\
& =-2 \bar{C}_{T}\left(1-x_{R}^{2}\right)^{-1} \int_{x_{R}}^{1} K^{-1}(\alpha) x^{2} \mathrm{~d} x
\end{aligned}
$$

where $K(\alpha)$ is the aerodynamic efficiency of the blade section, and no additional restrictions must be considered. The minimization of equation (27) gives rise to

$$
\frac{\mathrm{d}}{\mathrm{d} \alpha}\left(\frac{1}{K}\right)=0
$$

Result (28) implies that the inverse of the aerodynamic efficiency must be minimum, and therefore all the blade sections must operate at the optimum 
angle of attack, that is $\alpha=\alpha_{\mathrm{opt}}$, which is the traditional result for the OR. The optimum solidity can be written as

$$
\sigma(x)=\frac{4 \bar{C}_{T}}{C_{l, \mathrm{opt}}\left(1-x_{R}^{2}\right) x}
$$

where $C_{l, \mathrm{opt}}=C_{l}\left(\alpha_{\mathrm{opt}}\right)$. The optimum pitch angle distribution is

$$
\theta(x)=\alpha_{\mathrm{opt}}+\frac{1}{x} \sqrt{\frac{\bar{C}_{T}}{2\left(1-x_{R}^{2}\right)}}
$$

and using equations (4) and (5), the induced power coefficient results are equal to equation (24) and the profile power coefficient, as a function of the thrust coefficient and the maximum aerodynamic efficiency, $K_{\max }$, is

$$
C_{Q_{0}}=\frac{2 \bar{C}_{T}\left(1-x_{R}^{3}\right)}{3 K_{\max }\left(1-x_{R}^{2}\right)}
$$

where $\quad K_{\max }=C_{l, \mathrm{opt}} / C_{\mathrm{d}, \mathrm{opt}} \quad$ and $\quad C_{\mathrm{d}, \mathrm{opt}}=C_{d}\left(\alpha_{\mathrm{opt}}\right)$. Observe that these results depend only on the values of $C_{l, 0 \mathrm{ot}}, \alpha_{\mathrm{opt}}$ and $K_{\max }$, and therefore no assumptions are required on the functional dependencies $C_{l}(\alpha)$ and $C_{d}(\alpha)$.

\subsection{Minimum power coefficient rotor without root-tip losses}

The MPR is a rotor which minimizes the power coefficient, $C_{Q}$, for a given thrust coefficient, $\bar{C}_{T}$. In this case the control functions are again, as for the OR, $X_{1}(x)=\lambda_{i}(x), X_{2}(x)=\alpha(x)$ and $X_{3}(x)=\sigma(x)$, but the function $L$ is now

$$
L=\frac{\sigma}{2} x^{2}\left[-\lambda_{i} C_{l}(\alpha)+x C_{d}(\alpha)\right]
$$

The value of the Lagrange multiplier, $v$, results in $v=\mu-\lambda_{i}+x / K(\alpha)$ and equation (18) yields the following expression

$$
4 x^{2} \lambda_{i}^{2} \frac{\mathrm{d}}{\mathrm{d} \alpha}\left[\frac{1}{K(\alpha)}\right]=0
$$

which, assuming that $4 x^{2} \lambda_{i}^{2} \neq 0$, implies equation (28). Thus it is shown that as well as in the case of the OR, the MPR operates at the angle of attack that maximizes the aerodynamic efficiency. However, in the case of the MPR the following expression for the induced velocity is obtained

$$
\lambda_{i}=\frac{2}{3}\left(\mu+\frac{x}{K_{\max }}\right)
$$

which indicates that the induced velocity is a linear function of $x$. Using equation (34) in equation (10) the value for $\mu$ is obtained and the induced velocity results in

$$
\lambda_{i}=\frac{12\left(1-x_{R}^{2}\right) x+8\left(x_{R}^{3}-1\right)-A}{18\left(1-x_{R}^{2}\right) K_{\max }}
$$

with $A$ given by

$$
\begin{aligned}
& A= \\
& 2 \sqrt{2} \sqrt{-1+9 x_{R}^{2}-16 x_{R}^{3}+9 x_{R}^{4}-x_{R}^{6}+\frac{81 \bar{C}_{T} K_{\max }^{2}}{4}\left(1-x_{R}^{2}\right)}
\end{aligned}
$$

The optimum solidity can be expressed as

$$
\sigma(x)=\frac{2}{81} \frac{\left[12 x\left(x_{R}^{2}-1\right)+8\left(1-x_{R}^{3}\right)+A\right]^{2}}{K_{\max }^{2} C_{l, \mathrm{opt}}\left(1-x_{R}^{2}\right)^{2} x}
$$

and finally, the optimum pitch angle distribution for the MPR is given by

$$
\theta(x)=\alpha_{\text {opt }}-\frac{12 x\left(x_{R}^{2}-1\right)+8\left(1-x_{R}^{3}\right)+A}{18 K_{\max } x}
$$

It should be remarked that equation (38) is also a hyperbolic function of the dimensionless radial position, as well as in the ITR and OR cases. Using the above results in equations (4) and (5), the expressions that define the induced and profile power coefficients as a function of both the thrust coefficient and the maximum aerodynamic efficiency, can be expressed as

$$
C_{Q_{i}}=\frac{1}{14580} \frac{\sum_{i=0}^{9} h_{i} x_{R}^{i}}{K_{\max }^{3}\left(1-x_{R}^{2}\right)^{2}}
$$

and

$$
C_{\mathrm{Q}_{0}}=\frac{1}{1215} \frac{\sum_{i=0}^{8} g_{i} x_{R}^{i}}{K_{\max }^{3}\left(1+x_{R}\right)\left(1-x_{R}^{2}\right)}
$$

where the functions $h_{i}$ and $g_{i}$ are

$$
\begin{aligned}
& h_{0}=64+120 A+5 A^{3}, h_{1}=0, h_{2}=-1728-1080 A, \\
& h_{3}=6720+1920 A, h_{4}=-12096-1080 A, h_{5}=12096, \\
& h_{6}=-(6720-120 A), h_{7}=1728, h_{8}=0, h_{9}=-64
\end{aligned}
$$

and

$$
\begin{aligned}
& g_{0}=g_{1}=32-10 A+5 A^{2}, g_{2}=-112+80 A+5 A^{2}, \\
& g_{3}=-352-80 A, g_{4}=800+10 A, g_{5}=-352+10 A, \\
& g_{6}=-112, g_{7}=g_{8}=32
\end{aligned}
$$

As in the case of the OR, it should be pointed out that results (35) and (37) to (40), depend only on the prescribed $\bar{C}_{T}, C_{l, \mathrm{opt}}, \alpha_{\mathrm{opt}}$, and $K_{\max }$ values, and 
therefore no assumptions are required on the functional dependencies $C_{l}(\alpha)$ and $C_{d}(\alpha)$.

If $K_{\max }>1$ is assumed, equation (35) can be expanded in terms of $1 / K_{\max }$ yielding

$$
\lambda_{i} \approx-\sqrt{\frac{\bar{C}_{T}}{2\left(1-x_{R}^{2}\right)}}+\left[\frac{2}{3} x-\frac{4\left(1-x_{R}^{3}\right)}{9\left(1-x_{R}^{2}\right)}\right] \frac{1}{K_{\max }}+O\left(\frac{1}{K_{\max }^{2}}\right)
$$

Expression (43) shows that when the maximum aerodynamic efficiency tends to high values, the induced velocity which minimizes the power coefficient tends to the value corresponding to the traditional ITR and OR, expression (22). In other words, the induced velocity of the MPR is equal to the one that minimizes the induced power coefficient, plus a linear function of the dimensionless blade span position of order $1 / K_{\max }$. It must be noted that this correction is of the same order as the minimum order retained in the linearized equations of the BEMT, that is $C_{d}$.

\subsection{Optimization with tip-root losses}

The BEMT model applied in sections 3.1 to 3.3 does not include root or tip losses in the optimization process. Therefore, the optimum geometries obtained are likely to present unrealistic predictions of the rotor performances. In order to obtain geometries closer to the real optima, in this section, the OR and MPR including tip and root losses function defined in equation (2) are obtained. In what follows, the acronyms ORL and MPRL are used to identify the OR and MPR with root and tip losses.

In the case of the ORL, the optimization problem is, by definition, solved again in two steps. First the condition of minimum induced power coefficient, i.e. $L$ is given by equation (20), is applied. The consideration of the tip and root losses in the optimization requires the inclusion of an appropriate model for $F\left(\lambda_{i}\right) \neq 1$ (like the one presented in equation (2)) in equations (9) and (17). Now, solving equation (9) for $\sigma(x)$, (19) for $v(x)$ and substituting the obtained values in equation (17), a new equation for $\lambda_{i}(x)$ is obtained with the form

$$
2 \mu F\left(\lambda_{i}\right)-\lambda_{i}^{2} \frac{\mathrm{d} F\left(\lambda_{i}\right)}{\mathrm{d} \lambda_{i}}-\lambda_{i}\left[3 F\left(\lambda_{i}\right)-\mu \frac{\mathrm{d} F\left(\lambda_{i}\right)}{\mathrm{d} \lambda_{i}}\right]=0
$$

which, together with the two-point boundary-value problem (10), allows one to numerically obtain a solution for $\mu$ and $\lambda_{i}(x)$. It should be remarked that, as will be shown in section 4 , equation (44) yields a function $\lambda_{i}(x)$ that varies strongly with $x$ at the root and tip blade regions.
In a second step, the profile torque, $C_{Q_{0}}$, is minimized. As in subsection 3.2, in this minimization problem, the angle of attack is the only control function, and therefore the first identity in equation (27) also applies here, yielding, in this case, the functional

$$
\begin{aligned}
J[\alpha(x)] & =-\int_{x_{R}}^{1} \frac{\sigma}{2} x^{3} C_{d}(\alpha) \mathrm{d} x \\
& =-\int_{x_{R}}^{1} 4 \lambda_{i}^{2} F\left(\lambda_{i}\right) K^{-1}(\alpha) x^{2} \mathrm{~d} x
\end{aligned}
$$

whose minimization gives rise to equation (28), meaning that the blade sections must operate at their optimum angle of attack, $\alpha_{\text {opt }}$ and therefore $C_{l}(\alpha)=C_{l, \text { opt }}, C_{d}(\alpha)=C_{d, \text { opt }}$ and $K(\alpha)=K_{\text {max. }}$ From equation (9) the local solidity is expressed by

$$
\sigma(x)=\frac{8 \lambda_{i}^{2} F\left(\lambda_{i}\right)}{x C_{l, \mathrm{opt}}}
$$

and the pitch angle, $\theta(x)$, is

$$
\theta(x)=\alpha_{\text {opt }}-\frac{\lambda_{i}(x)}{x} .
$$

Observe that now neither $\sigma(x)$ nor $\theta(x)$ are hyperbolic, as in the case of the traditional OR, since $\lambda_{i}(x)$ and $F\left(\lambda_{i}\right)$ introduce additional dependencies on the radial position, $x$. The induced and profile torque coefficients are obtained from expressions (4) and (5).

In the case of the MPRL, when the tip and root losses are considered, equations (9) and (17) are formally identical to the case of previously developed ORL. The function $L$ is now given by (32). Solving equation (9) for $\sigma(x)$ and equation (19) for $\nu(x)$, and substituting both results in equation (18), gives

$$
4 x^{2} F\left(\lambda_{i}\right) \lambda_{i}^{2} \frac{\mathrm{d}}{\mathrm{d} \alpha}\left[\frac{1}{K(\alpha)}\right]=0
$$

where it has been considered that $C_{d}(\alpha)=C_{l}(\alpha) / K(\alpha)$. Observe that equation (48) indicates again that the blade sections must operate at their optimum angle of attack. After imposing the condition $\alpha=\alpha_{\text {opt }}$ and the solutions previously obtained for $\sigma(x)$ and $v(x)$, equation (17) results in

$$
\begin{aligned}
& \left(3 \lambda_{i}-2 \frac{x+\mu K_{\max }}{K_{\max }}\right) F\left(\lambda_{i}\right) \\
& \quad+\lambda_{i}\left(\lambda_{i}-\frac{x+\mu K_{\max }}{K_{\max }}\right) \frac{\mathrm{d} F\left(\lambda_{i}\right)}{\mathrm{d} \lambda_{i}}=0
\end{aligned}
$$

The solution of equation (49) along with the twopoint boundary-value problem (10) allows one to numerically obtain the value of $\mu$ and the radial distribution of the induced velocity $\lambda_{i}(x)$. Finally, the induced and profile torque coefficients are obtained from expressions (4) and (5). 


\section{DISCUSSION}

In what follows the results of the five optimization problems above analysed, ITR, OR, MPR, ORL, and MPRL, are presented. In all cases the number of blades considered is $b=3$ and the root cutout is $x_{R}$ $=0.1$. The five rotors have been determined to obtain the same given thrust coefficient, $\bar{C}_{T}=0.005$. The airfoil for all the rotors is a NACA-0012. Following the data presented in reference [22] for a Reynolds number of $8 \times 10^{4}$, the slope of the lift coefficient of a linear fit is $C_{l, \alpha}=5.73$, and the drag coefficients of a parabolic fit are $\delta_{0}=0.0150, \delta_{1} \approx 0.0$ and $\delta_{2}=1.3709$. Using these values of the lift and drag coefficients, the maximum aerodynamic efficiency is $K_{\max }=20.0$ and the optimum lift coefficient is $C_{l, \mathrm{opt}}=0.59$, which correspond to an optimum angle of attack $\alpha_{\mathrm{opt}}=5.99$.

The ITR has one degree of freedom which is the value of the uniform solidity. In order to compare the five optimum rotors the constant solidity of the ITR has been chosen as the solidity, which minimizes the power coefficient. In order to achieve this condition, the solidity which minimizes the profile power coefficient is obtained from the expression $\mathrm{d}_{Q_{0}} /$ $\mathrm{d} \sigma=0$. Taking into account expressions (24) and (25), and using the value of the angle of attack at the tip, that is $\alpha=4 \bar{C}_{T}\left[\left(1-x_{R}^{2}\right) \sigma C_{l, \alpha}\right]^{-1}$, the following optimum value of solidity is obtained

$$
\sigma=\frac{4 \sqrt{2 C}_{T}}{C_{l, \mathrm{opt}}\left(1-x_{R}^{2}\right) \sqrt{1+x_{R}^{2}}}
$$

When the above-mentioned values are used, the solidity of the ITR which minimizes the power coefficient is $\sigma=0.047$.

The radial distributions of dimensionless induced velocity are presented in Fig. 1. In the cases of the traditional ITR and OR, an equal constant value along the blade span, given by expression (22), is presented, whereas for the MPR, the linear distribution given by equation (35) is shown. Observe, that the MPR compared with the ITR and OR, presents a value of $\lambda_{i} 36$ per cent higher in absolute value (more downwash) at the blade root and 24 per cent lower in absolute value (less downwash) at the tip. The difference is important in this case owing to the low value of the maximum aerodynamic efficiency $\left(K_{\max }=20\right)$ as is shown in equation (43).

When root and tip losses are considered in the optimization process, the respective uniform and linear distributions of $\lambda_{i}$ of the OR and the MPR are modified significantly. The downwash decreases in the regions close to the root and tip, whereas it increases at the middle of the blade, keeping, in this blade region, approximately, uniformity in the case of the ORL, and linearity in the case of the MPRL.

Figure 2 shows the solidity of the five rotors. As already mentioned, when neither root nor tip losses are considered, both the OR and MPR exhibit a hyperbolic dependency, but the OR presents lower solidity at inner sections of the blade, while the MPR shows a lower solidity at the outer sections. Root and tip losses, in the ORL and MPRL, make the local solidity tend to zero both at the root and the tip. The maximum local solidity is significantly decreased when root losses are considered. Hence, in the case of the OR compared to the ORL, this reduction is from

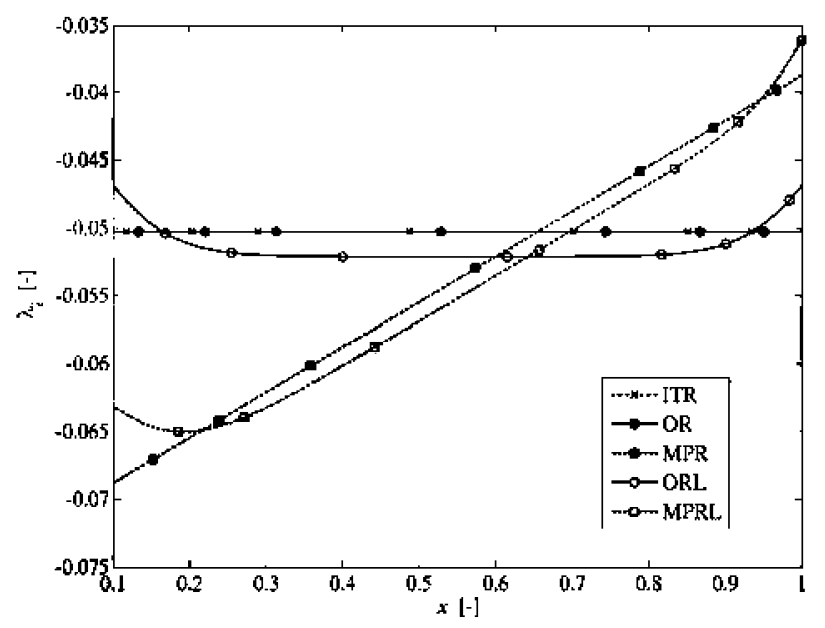

Fig. 1 Optimum distributions of induced velocity for the ITR with solidity $\sigma=0.047$, OR, MPR, ORL, and MPRL for a given thrust coefficient $\bar{C}_{T}=0.005$ and NACA 0012 airfoils at Reynolds number of $8 \times 10^{4}$. The number of blades is $b=3$ and the root cutout is $x_{R}=0.1$

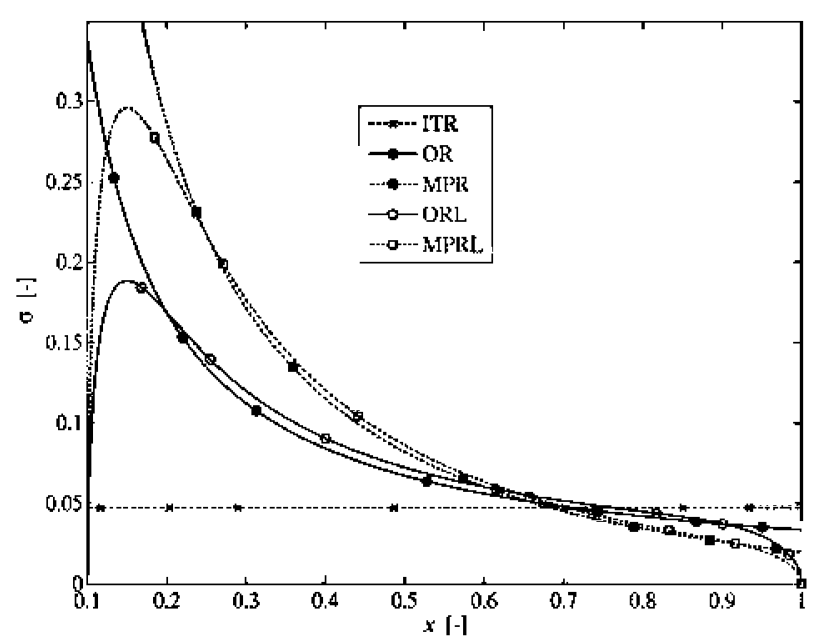

Fig. 2 Optimum distributions of solidity for the ITR $(\sigma=0.047)$, OR and MPR, ORL and MPRL for the conditions reported in Fig. 1 
$\sigma_{\text {max }}=\sigma(0.1) \approx 0.37$ down to $\sigma_{\max }=\sigma(0.15) \approx 0.18$. In the case of the MPR compared with the MPRL, this reduction is from $\sigma_{\max }=\sigma(0.1) \approx 0.6$ down to $\sigma_{\max }=$ $\sigma(0.18) \approx 0.295$.

The reduction of the maximum value of the optimum solidity distribution as well as the change of its radial location to higher $x$ values, is a beneficial effect of root losses, from the point of view of the manufacturability of the rotor. As an example, the layouts of the four rotors OR, ORTL, MPR, and MPRTL are depicted in Fig. 3, for the conditions defined at the

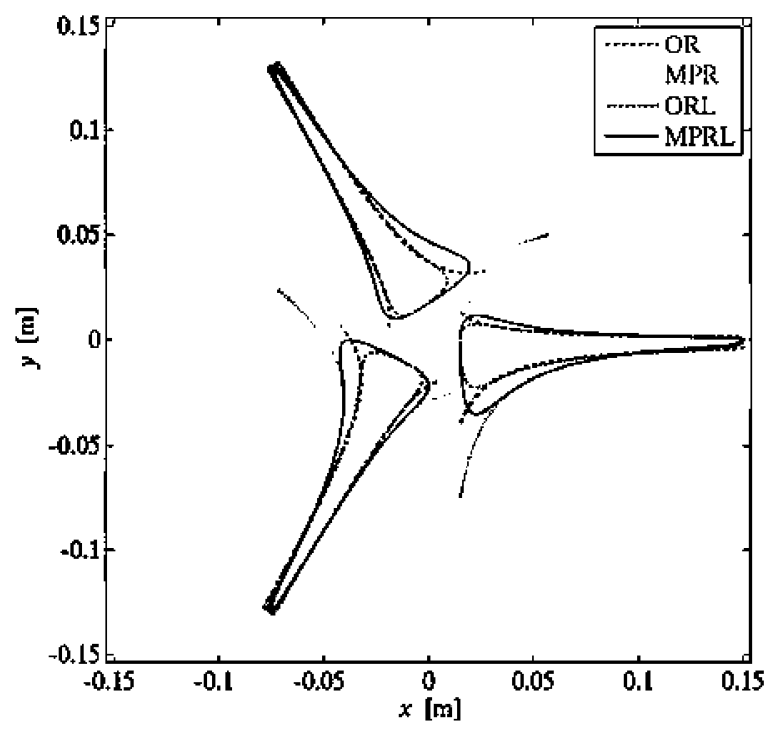

Fig. 3 Geometry of a three blade for the cases OR, MPR, ORL, and MPRL for the conditions reported in Fig. 1 and radius of $0.15 \mathrm{~m}$

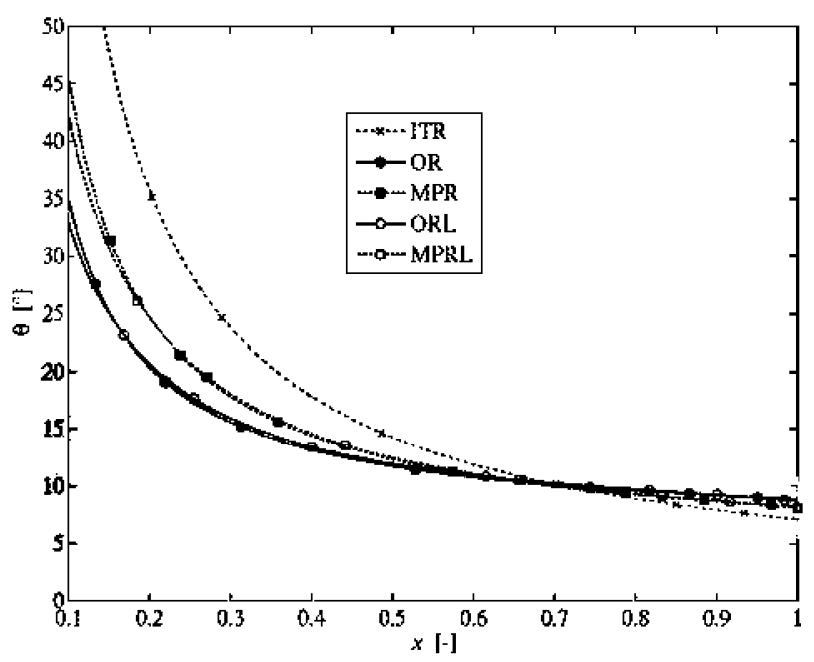

Fig. 4 Optimum distributions of pitch angle for the ITR $(\sigma=0.047)$, OR and MPR, ORL and MPRL for the conditions reported in Fig. 1 beginning of this section and for a rotor with radius $R=0.15 \mathrm{~m}$.

Figure 4 shows the pitch angle distributions. The value corresponding to the ITR at the inner section is remarkably higher compared to both the OR and the MPR while at the outer sections this tendency is reversed. The inclusion of root and tip losses does not significantly change the radial distribution of the pitch angle. In the case of the OR, MPR and their corresponding counterparts with root and tip losses, ORL and MPRL, the maximum value of the pitch angle is less than $45^{\circ}$, which is perfectly realizable.

The aerodynamic efficiency is shown in Fig. 5. Only the ITR presents a non-constant distribution of $K$ since the angle of attack is not constant. In fact, the angle of attack of this rotor shows a hyperbolic radial dependency. In the case of the OR, MPR, ORL, and MPRL, the aerodynamic efficiency corresponds to the maximum value as was obtained in equation (27) for the OR and ORL and in equations (33) and (48) for the MPR and MPRL respectively. It must be remarked that the authors consider that $\alpha_{\text {opt }}, C_{l, o p t}$, and $K_{\max }$ are constant along the blade span. Obviously this is not exactly true since the local Reynolds number based on the local velocity and the chord of the blade section is not constant. However, within the scope of this paper this hypothesis is considered accurate enough.

In order to illustrate the differences found in the performances of the five rotors, the radial distributions of thrust coefficient are shown in Fig. 6. The five $\mathrm{d} C_{T} / \mathrm{d} x$ distributions have the same integral value $\bar{C}_{T}=0.005$. The ITR and OR present the known linear distribution. The MPR presents a

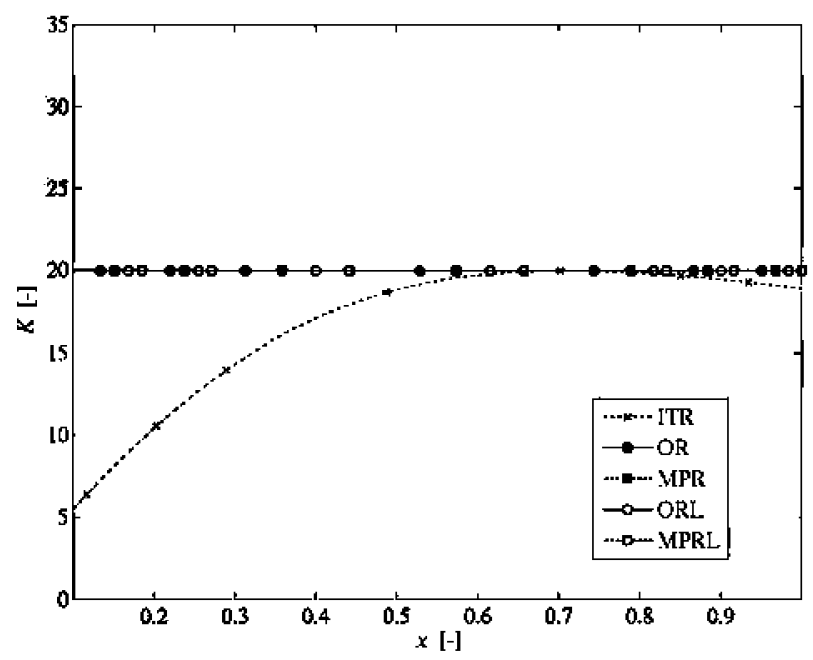

Fig. 5 Optimum distributions of aerodynamic efficiency for the ITR ( $\sigma=0.047)$, OR and MPR, ORL and MPRL for the conditions reported in Fig. 1 


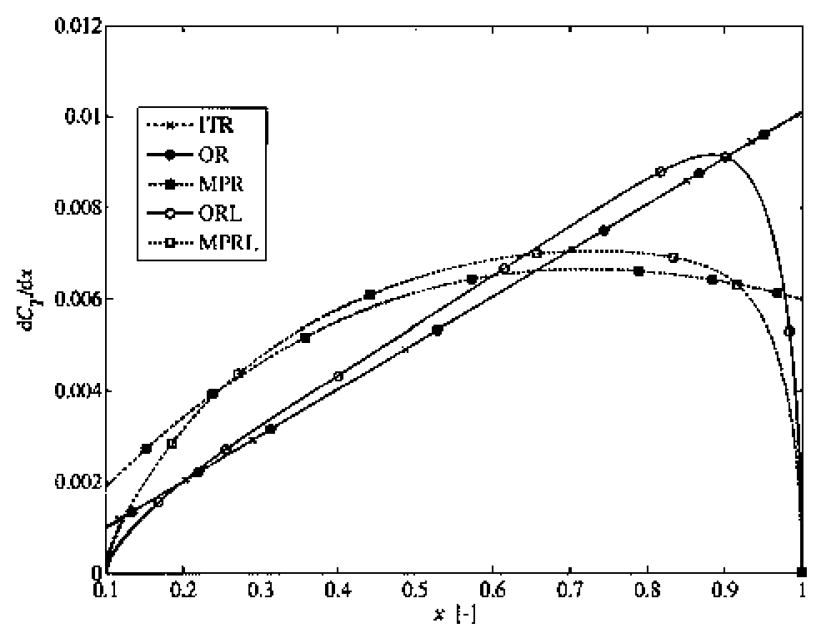

Fig. 6 Radial distributions of thrust coefficient for the ITR $(\sigma=0.047)$, OR and MPR, ORL and MPRL for the conditions reported in Fig. 1

much more uniform distribution along the blade span, which can be interpreted as a design advantage, since the bending moments at the root will be less than in the ITR and OR. Root and tip losses make the values of the distribution zero at the root and at the tip. Again, in the MPRL, the inner blade sections contribute more to the $C_{T}$ than in the ORL. The tendency in the outer blade sections is inverted. Observe that the maximum value of $\mathrm{d} C_{T} / \mathrm{d} x$ for the MPRL is reduced significantly and moves towards the root compared to the ORL. This characteristic is likely to mean an advantage in the structural design of the blade coupling at the root, probably giving rise to lighter blade designs. This goal is one of the four main challenges for the development of MAV identified in reference $[\mathbf{1}]$.

The induced power coefficient distribution is shown in Fig. 7. As is well known, the radial distribution of the induced power coefficient of the ITR and OR is a linear one. It is shown, however, that the induced power of the MPR is a non-linear distribution which, compared with the ITR and OR, is higher at the inner blade sections and lower at the outer sections. The MPR presents a much more uniform radial distribution of $\mathrm{d} C_{Q_{i}} / \mathrm{d} x$ compared with both the ITR and the OR. This result is similar to the one obtained for the distribution of thrust coefficient. Root and tip losses make the value of the induced torque distribution go to zero at the blade extremes. It is remarkable that the MPRL presents a much flatter radial distribution compared to the ORL. This characteristic dominates, as is shown below, the shape of the total torque coefficient distribution.

The profile power coefficient (Fig. 8) of the ITR is evidently higher than the profile power of the OR. In

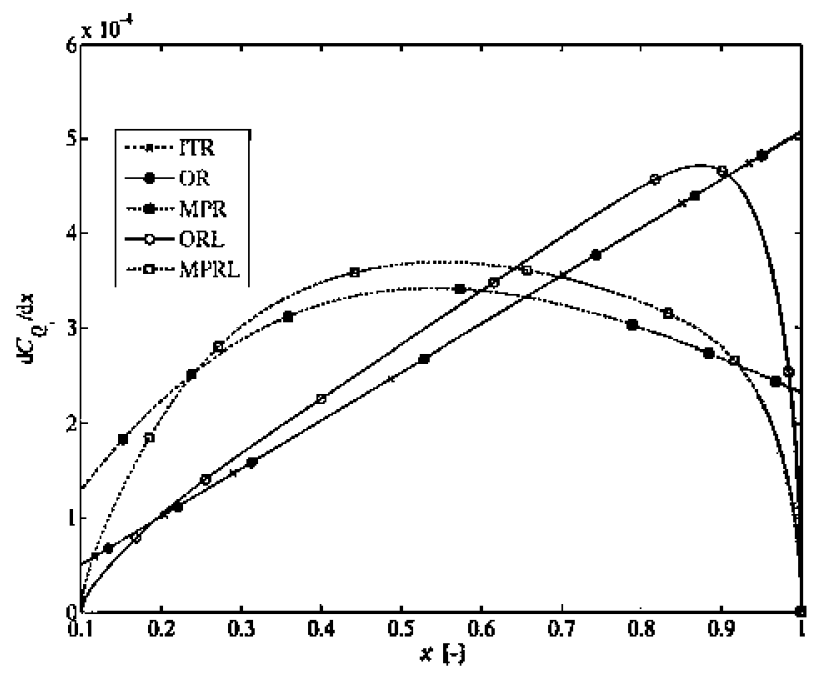

Fig. 7 Radial distributions of induced power coefficient for the ITR $(\sigma=0.047)$, OR and MPR, ORL and MPRL for the conditions reported in Fig. 1

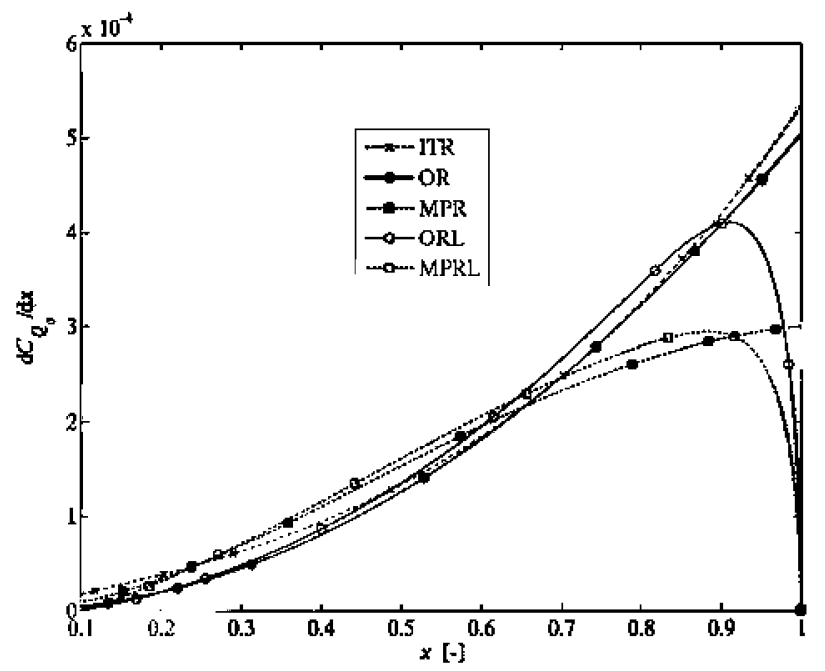

Fig. 8 Radial distributions of profile power coefficient for the ITR ( $\sigma=0.047)$, OR and MPR, ORL and MPRL for the conditions reported in Fig. 1

addition, the OR presents a lower profile power coefficient at the inner sections, while, at the outer sections, the MPR shows a lower profile power coefficient. The effect of the root and tip losses is similar in this case. As is expected, the distributions go to zero at both blade extremes for the ORL and the MPRL since $\sigma(x)$ does in both cases.

Finally, Fig. 9 shows the total power coefficient. It can be seen that the ITR power coefficient distribution is higher than that for both the OR and MPR. The MPR presents a flatter distribution of total torque coefficient. When root and tip losses are considered, the ORTL and MPRL distributions tend to zero at the 
blade extremes, as expected. The radial distribution for the MPRL is flatter, and the corresponding maximum value is less compared with that of ORL, and it is located at a significantly smaller radial position.

Table 1 summarizes the global power coefficients and shows the global value of induced, profile and total power coefficients for the five rotors. It is clear that both the ITR and OR present the minimum induced power while the MPR exhibits the lowest value of profile power. The reduction of profile power of the MPR is high enough to ensure that the MPR requires the minimum amount of total power, as expected. Root and tip losses increase the power consumption, but even considering this effect, the power saving of the MPRL compared to the ORL is about 1.7 per cent. As is analysed below, this power saving increases significantly for lower values of $\bar{C}_{T}$ and $K_{\max }$.

Figure 10 represents the ratio between the induced power coefficients of the MPR and OR as a function of the given thrust coefficient, $\bar{C}_{T}$, with the maximum aerodynamic efficiency, $K_{\max }$ as a parameter. The induced power coefficient ratio between the MPR and $\mathrm{OR}$ is, as expected, higher than one owing to the fact that the OR minimizes the induced power

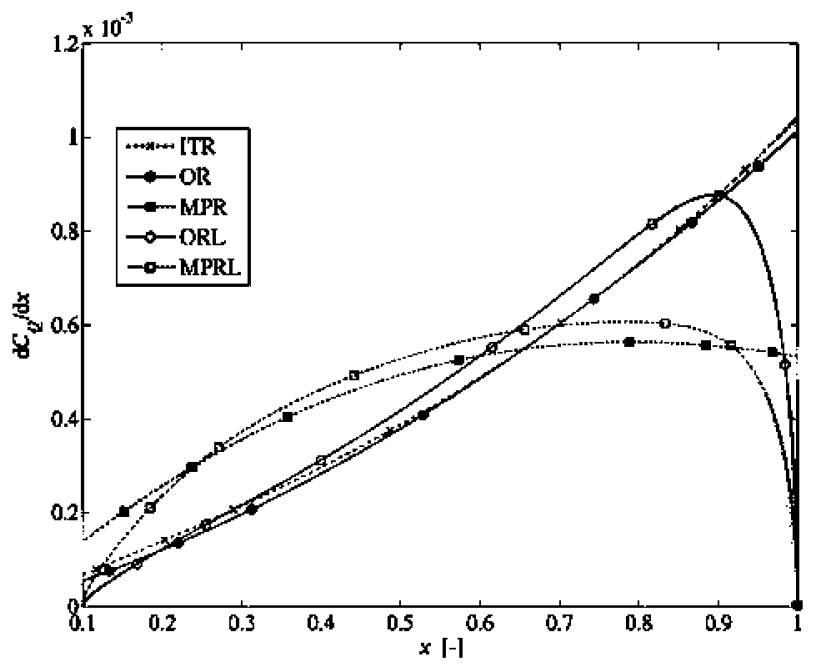

Fig. 9 Radial distributions of total power coefficient for the ITR ( $\sigma=0.047)$, OR and MPR, ORL and MPRL for the conditions reported in Fig. 1 coefficient and therefore the induced power of the OR is a minimum. However, the MPR presents an induced velocity which is not constant with respect to the dimensionless blade span position, therefore leading to an induced power coefficient which is not minimum. The ratio increases significantly as the values of $\bar{C}_{T}$ and $K_{\max }$ decrease. The value for this ratio is about 1.34 for $\bar{C}_{T}=0.002$ and $K_{\max }=10$ when neither root nor tip losses are considered. The difference is reduced when root and tip losses are taken into account. For high values of $K_{\max }$ the ratio is almost one.

The ratio of profile torque coefficient follows the opposite trend (Fig. 11). It is less than one, indicating that the profile torque coefficient is lower for the MPR compared to the OR. The reduction of $C_{Q_{0}}$ for the MPR reaches 32 per cent for $\bar{C}_{T}=0.002$ and $K_{\max }=$ 10. The effect of root and tip losses is to reduce this difference, hence the MPRL presents a 29 per cent reduction in $C_{Q_{0}}$ compared to the ORL. As in the case of the induced power coefficient, the differences between MPR and OR, and between the MPRL and

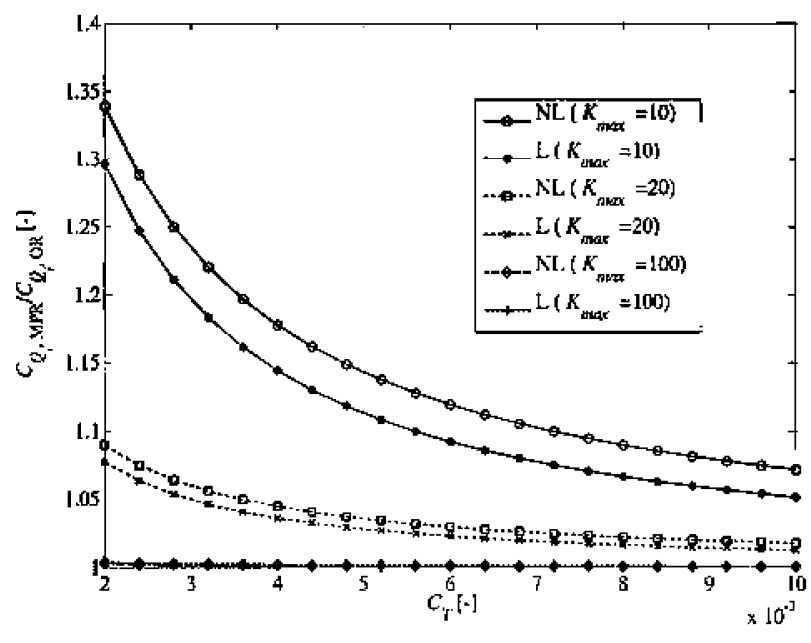

Fig. 10: Ratio of global induced power coefficients between MPR and OR without and with root-tip losses (NL and $\mathrm{L}$ respectively) as functions of global thrust coefficient for several values of maximum aerodynamic efficiency. The number of blades is $b=3$ and the root cutout is $x_{R}=0.1$

Table 1 Global power coefficients for the ITR with solidity $\sigma=0.047$, OR, MPR, ORL, and MPRL for a given thrust coefficient $\bar{C}_{T}=0.005$ and NACA 0012 airfoils at Reynolds number of $8 \times 10^{4}$. The number of blades is $b=3$ and the root cutout is $x_{R}=0.1$

\begin{tabular}{lllll}
\hline & ITR & OR & MPR & ORL \\
\hline$C_{Q_{i}}$ & $2.512 \times 10^{-4}$ & $2.512 \times 10^{-4}$ & $2.602 \times 10^{-4}$ & $2.580 \times 10^{-4}$ \\
$C_{Q_{0}}$ & $1.777 \times 10^{-4}$ & $1.682 \times 10^{-4}$ & $1.503 \times 10^{-4}$ & $2.653 \times 10^{-4}$ \\
$C_{Q}$ & $4.289 \times 10^{-4}$ & $4.194 \times 10^{-4}$ & $4.105 \times 10^{-4}$ & $1.647 \times 10^{-4}$ \\
\hline
\end{tabular}




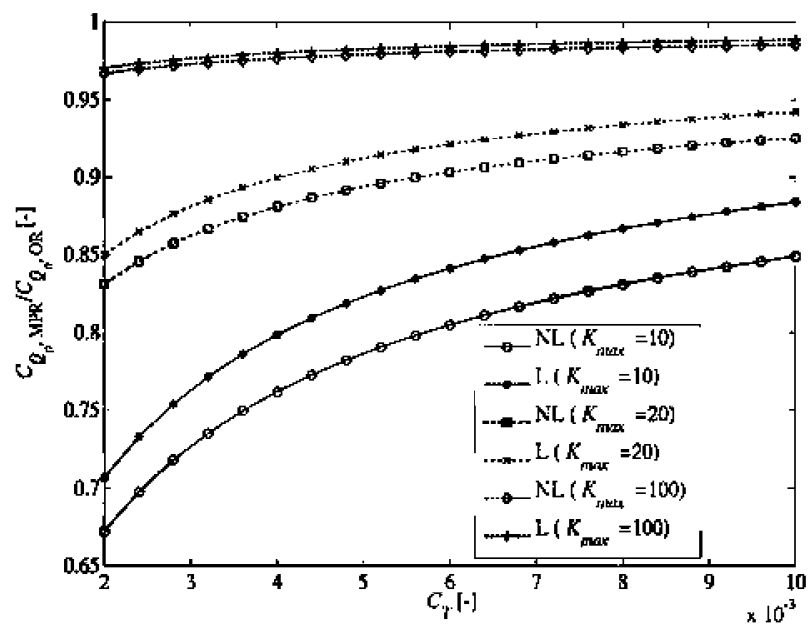

Fig. 11 Ratio of global profile power coefficients between MPR and OR without and with root-tip losses (NL and $\mathrm{L}$ respectively) as functions of global thrust coefficient for several values of maximum aerodynamic efficiency. The number of blades is $b=3$ and the root cutout is $x_{R}=0.1$

ORL are less as $\bar{C}_{T}$ and $K_{\max }$ increase, thus the differences in $C_{Q_{0}}$ go down to 2 per cent for $\bar{C}_{T}=0.01$ and $K_{\max }=100$.

The ratios of the total power coefficient of the MPR to the one of the OR and their counterparts with root and tip losses are presented in Fig. 12. The overall behaviour of the results shows that the OR does not minimize the total power coefficient. The MPR evidently requires lower power than the $\mathrm{OR}$, because the reduction of the profile power coefficient of the MPR outweighs the increase of its induced power coefficient when it is compared to the OR. It is also worth observing that the differences between the MPR and OR are higher when $\bar{C}_{T}$ and $K_{\max }$ are lower, yielding a remarkable saving of power consumption. The effect of root and tip losses is to slightly decrease the reduction in power consumption of the MPRL compared with the ORL. The reduction in $C_{Q}$ reaches a value of 10 per cent for $\bar{C}_{T}=0.002$ and $K_{\max }=10$. The saving in $C_{Q}$ for high values of $\bar{C}_{T}$ and $K_{\max }$ is almost negligible.

Regarding the figure of merit (FM), the relative difference between the MPR and OR and their corresponding counterparts including root and tip losses are shown in Fig. 13. It can be observed that the relative difference of the FM increases, not only when the maximum aerodynamic efficiency is low, but, also, when the thrust coefficient is low. Therefore the situation in which the OR and MPR differ more, corresponds to low thrust coefficient and low maximum aerodynamic efficiency values. As is mentioned in section 1, this situation usually

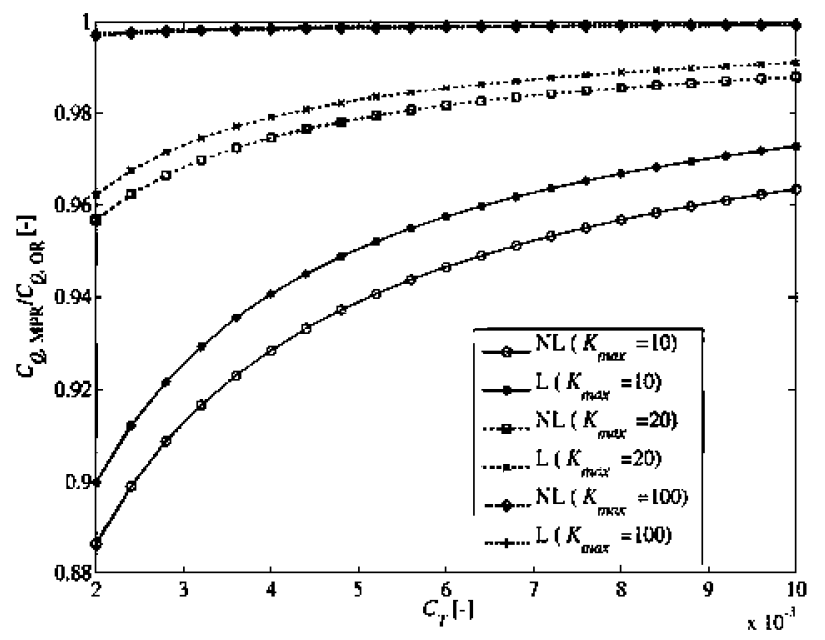

Fig. 12 Ratio of global total power coefficients between MPR and OR without and with root-tip losses (NL and $\mathrm{L}$ respectively) as functions of global thrust coefficient for several values of maximum aerodynamic efficiency. The number of blades is $b=3$ and the root cutout is $x_{R}=0.1$

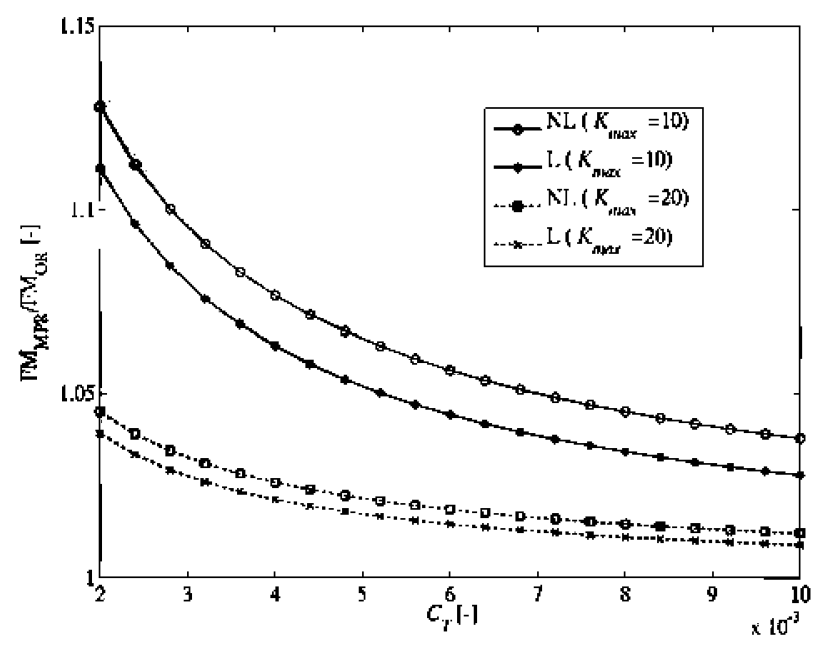

Fig. 13 Relative difference of FM between MPR and OR without and with root-tip losses (NL and $L$ respectively) as functions of global thrust coefficient for several values of maximum aerodynamic efficiency. The number of blades is $b=3$ and the root cutout is $x_{R}=0.1$

appears in the design of rotary wing MAV. Root and tip losses moderate the improvement of the FM of the MPRL compared to the ORL, reaching an increment of 10 per cent for $\bar{C}_{T}=0.002$ and $K_{\max }=10$.

Figure 14 shows the comparison for the FM of the OR, MPR, ORL, and MPRL with experimental results presented in reference [23] with respect to the thrust coefficient. The experimental results obtained from reference $[\mathbf{2 3}]$ correspond to two rotors, both with a 


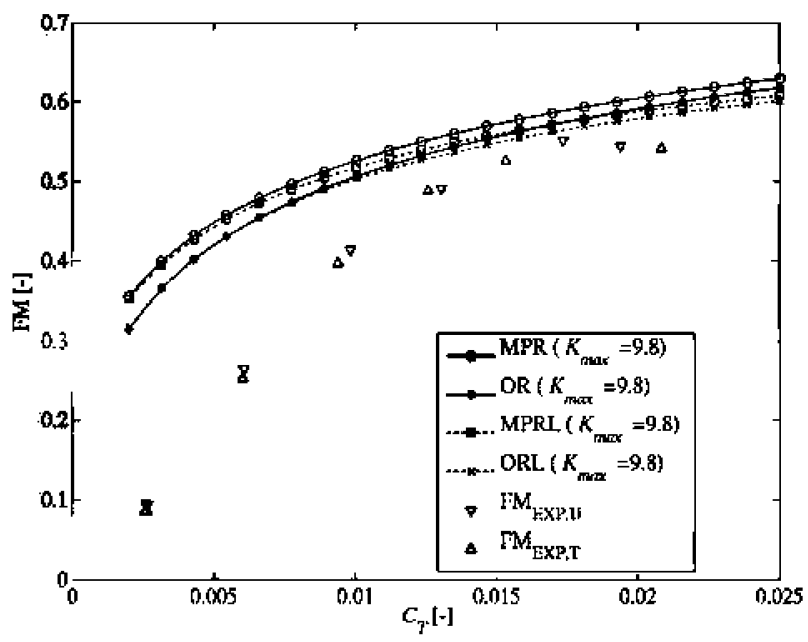

Fig. 14 Comparison of FM for the MPR, OR, MPRL, and ORL with respect to experimental data, of a MAV rotor blade at Reynolds number of $27 \times 10^{3}$ where $\mathrm{FM}_{\mathrm{EXP}, T}$ stands for experimental FM of twisted blade, $\mathrm{FM}_{\mathrm{EXP}, U}$ stands for experimental FM of untwisted blade, after reference [21]

solidity of $\sigma=0.118$, one untwisted, and the second one with a-10 degrees linear twist. The airfoil of the blade is an 8 per cent camber flat plate and the aerodynamic lift and drag coefficients have been determined using BEMT along with the experimental data of the untwisted blade, as is reported in reference [23]. The maximum aerodynamic efficiency is $K_{\max }=9.8$ and the optimum angle of attack $\alpha_{\mathrm{opt}}=9.8$ degrees. Using these values, the OR, ORL, MPR and MPRL are completely defined. As can be seen, the lower the value of the thrust coefficient, the greater the difference between the OR and MPR with the experimental data. The maximum aerodynamic efficiency is about 10 , hence, this value is low enough to yield an important difference between the FM of the OR with respect to the MPR, which represents the upper bound. As stated in reference [23], the differences between twisted and untwisted configurations are not very important. However, as also indicated, the profile power plays a fundamental role in power losses and therefore the MPR shows the importance of defining not only the appropriate twist distribution, but also the chord distribution, in order to obtain the maximum aerodynamic performance in hover. Observe that when root and tip losses are considered, both, the ORL and MPRL performances are closer to the experimental results.

Figure 15 shows, depending upon the value of the thrust coefficient, that the improvement in the FM is about four times the experimental value at low thrust coefficient values, while at high values is about 10 per cent higher.

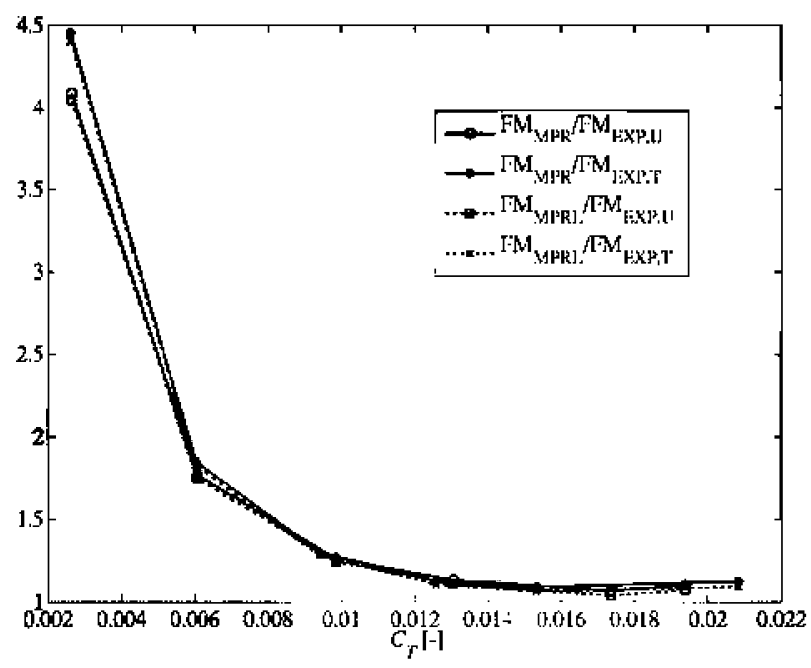

Fig. 15 Ratio of FM between MPRL and experimental values of an actual MAV rotor, for a Reynolds number of $27 \times 10^{3}$. FM $\mathrm{EXP}, T_{T}$ stands for experimental FM of twisted blade, $\mathrm{FM}_{\mathrm{EXP}, U}$ stands for experimental FM of untwisted blade, after reference [21]

\section{CONCLUSIONS}

In this article calculus of variations has been used to define five optimum rotors. Two of them correspond to the well-known ITR and OR. The third rotor analysed in this paper is the rotor which minimizes the total power coefficient, MPR.

An interesting result is that the MPR does not produce a constant induced velocity along the blade span. Moreover, the induced velocity radial distribution is a linear one. As far as the authors know, the MPR obtained in the present paper has not been reported in the literature before. Also, the derivation from a calculus of variation approach of the wellknown ITR and OR has not been reported before.

The results presented herein are valuable and interesting in themselves for two reasons. First, from an educational point of view, the use of calculus of variations is an excellent tool to show students a rigorous mathematical demonstration of the solidity, twist, and induced velocity distributions of the ITR, OR, and MPR. Second, calculus of variations can be used with more complex simulation models and the final optimization problems will also be more complicated to deal with. Evidently, in this case, the problems should be tackled with numerical methods in order to obtain the corresponding solution.

The MPR tends to the OR when the aerodynamic efficiency is high enough. This behaviour opens an important practical application for the MPR owing to the low values of aerodynamic efficiency that rotary wing MAV exhibit. 
When both root and tip losses are included in the optimization process, the optimum solidity is significantly changed at the blade root and tip, leading to more realizable rotors. For maximum aerodynamic efficiency of about 10, the ratio between the FM of the MPRL and that of the ORL can be about 3 per cent for high values of thrust coefficient, about 0.01 , and of 11 per cent for thrust coefficient about 0.002 .

Finally, and considering the blade shape of the MPRL obtained, its implementation to MAV could lead to new designs in this leading technological field. Of course, a trade-off between aerodynamic performance and simple manufacturing of MAV rotor blades would be encountered. The MPRL obtained in this paper should stimulate micro air vehicles manufacturing processes that permit the production of more complex geometry rotor blades.

\section{FUNDING}

This research received no specific grant from any funding agency in the public, commercial, or notfor-profit sectors.

\section{(C) Authors 2011}

\section{REFERENCES}

1 Pines, D., Bohorquez, F., and Samuel, P. D. Challenges facing future micro-air-vehicle development. J. Aircraft, 2006, 43(2), 290-305.

2 Tsuzuki, N., Sato, S., and Abe, T. Design guidelines of rotary wings in hover for insect-scale micro air vehicle applications. J. Aircraft, 2007, 44(1), 252-263.

3 Ganguli, R. Survey of recent developments in rotorcraft design optimization. J. Aircraft, 2004, 41(3), 493-510.

4 Nadarajah, S. K. and Tatossian, C. Multi-objective aerodynamic shape optimization for unsteady viscous flows. Optimization and Engng, 2010, 11 (1), 67-105.

5 Le Pape, A. and Beaumier, P. Numerical optimization of helicopter rotor aerodynamic performance in hover. Aerospace Sci. Technol., 2005, 9(3), 191-201.

6 Rand, O., Khromov, V., and Peyran, R. J. Minimuminduced power loss of a helicopter rotor via circulation optimization. J. Aircraft, 2004, 41 (1), 104-109.

7 Leishman, J. G. and Ananthan, S. An optimum coaxial rotor system for axial flight. J. Am. Helicopter Soc., 2008, 53(4), 366-381.

8 Ganguli, R., Jehnert, B., Wolfram, J., and Vrsmann, P. Optimal location of centre of gravity for swashplateless helicopter UAV and MAV. Aircraft Engng Aerospace Technol., 2007, 79(4), 335-345.

9 Siva, C., Murugan, M. S., and Ganguli, R. Effect of uncertainty on helicopter performance predictions. Proc. IMechE, Part G: J. Aerospace Engineering, 2010, 224(5), 549-562.
10 Sunada, S., Sakaguchi, A., and Kawachi, K. Airfoil section characteristics at a low Reynolds number. J. Fluids Engng, 1997, 119, 129-135.

11 Bohorquez, F., Pines, D., and Samuel, P. D. Small rotor design optimization using blade element momentum theory and hover tests. J. Aircraft, 2010, 47(1), 268-283.

12 Lissaman, P. B. S. Low-Reynolds-number airfoils. $A$. Rev. Fluid Mechanics, 1983, 15, 223-239.

13 Vogel, S. Flight in drosophila. iii. aerodynamic characteristics of fly wings and wing models. J. Expl. Biol., 1967, 46, 431-443.

14 Carmichael, B. H. Low Reynolds number airfoil survey. NASA-CR-165803, November 1981.

15 Ramasamy, M., Leishman, J. G., and Lee, T. E. Flow field of a rotating-wing micro air vehicle. In Proceedings of the 62nd Annual Forum and Technology Display of the American Helicopter Society, Phoenix, Arizona, 2006, pp. 1-10.

16 Bohorquez, F., Samuel, P. D., Sirohi, J., Rudd, L., and Pines, D. Design, analysis and hover performance of a rotary wing micro air vehicle. J. Am. Helicopter Soc., 2003, 48(2), 80-90.

17 Tokaty, G. A. A history and philosophy of fluid mechanics, 1994 (Dover Publications).

18 Gessow, A. and Myers Jr, G. C. Aerodynamics of the helicopter, 1967 (Frederick Ungar Publishing).

19 Johnson, W. Helicopter theory, 1994 (Dover Publications).

20 Leishman, J. G. Principles of helicopter aerodynamics cambridge aerospace S, 2nd edn, 2006 (Cambridge University Press).

21 Bryson Jr., A. E. and Ho, Y. Applied optimal control, 1975 (Taylor \& Francis).

22 Miley, S. J. A catalog of low Reynolds number airfoil data for wind turbine applications. RFP-3387 VC-60, US Department of Energy Rockwell International, 1982.

23 Bohorquez, F., Rankins, F., Baeder, J. D., and Pines, D. Hover performance of rotor blades at low Reynolds numbers for rotary wing micro air vehicles. An experimental and CFD study. 2003, In Proceedings of AIAA 21st Applied Aerodynamics Conference.

\section{APPENDIX}

\section{Notation}

$b \quad$ number of blades

$C_{d} \quad$ drag coefficient

$C_{d, \mathrm{opt}}$ drag coefficient at which the aerodynamic

efficiency is maximum

$C_{l} \quad$ lift coefficient

$C_{l, \alpha} \quad$ lift coefficient slope

$C_{l, \text { opt }} \quad$ lift coefficient at which the aerodynamic efficiency is maximum

$C_{Q} \quad$ power coefficient

$C_{Q_{i}} \quad$ induced power coefficient 


$\begin{array}{ll}C_{Q_{0}} & \text { profile power coefficient } \\ C_{T} & \text { thrust coefficient } \\ \bar{C}_{T} & \text { given value of thrust coefficient } \\ F & \text { Root-tip losses function } \\ J & \text { functional } \\ J^{*} & \text { augmented functional } \\ K & \text { aerodynamic efficiency } \\ K_{\max } & \text { maximum aerodynamic efficiency } \\ L & \text { objective function } \\ n & \text { number of control functions } \\ X_{i} & \text { control function } \\ x & \text { dimensionless position along the blade } \\ x_{R} & \text { dimensionless position of the root cutout } \\ \alpha & \text { angle of attack } \\ \alpha_{\mathrm{opt}} & \text { angle of attack at which the aerodynamic } \\ & \text { efficiency is maximum } \\ \delta & \text { variation } \\ \delta_{0}, \delta_{1}, \delta_{2} & \text { profile drag parameters, } \\ \lambda_{i} & C_{d}=\delta_{0}+\delta_{1} \alpha+\delta_{2} \alpha^{2} \\ \text { dimensionless induced velocity }\end{array}$
$\mu \quad$ Lagrange multiplier
$v \quad$ Lagrange multiplier
$\sigma \quad$ rotor local solidity
$\theta \quad$ pitch angle

\section{Operators}

$\begin{array}{ll}\text { d } & \text { derivative } \\ \text { d } & \text { partial derivative }\end{array}$

\section{Acronyms}

BEMT blade element momentum theory

FM figure of merit

ITR ideal twist rotor

MAV micro air vehicle

MPR minimum power coefficient rotor

MPRL minimum power coefficient rotor with

root-tip losses

OR optimum rotor

ORL optimum rotor with root-tip losses 